Volume 70, Number 1, Pages 130-142(2021)

DOI: $10.31801 /$ cfsuasmas.780723

ISSN 1303-5991 E-ISSN 2618-6470

Received by the editors: August 14, 2020; Accepted: November 12, 2020

\title{
BEST PROXIMITY POINT THEORY ON VECTOR METRIC
} SPACES

\author{
Hakan SAHIN \\ Department of Mathematics, Faculty of Science and Arts, Amasya University, Amasya, \\ TURKEY
}

\begin{abstract}
In this paper, we first give a new definition of $\Omega$-Dedekind complete Riesz space $(E, \leq)$ in the frame of vector metric space $(\Omega, \rho, E)$ and we investigate the relation between Dedekind complete Riesz space and our new concept. Moreover, we introduce a new contraction so called $\alpha$-vector proximal contraction mapping. Then, we prove certain best proximity point theorems for such mappings on vector metric spaces $(\Omega, \rho, E)$ where $(E, \leq)$ is $\Omega$-Dedekind complete Riesz space. Thus, for the first time, we acquire best proximity point results on vector metric spaces. As a result, we generalize some fixed point results proved on both vector metric spaces and partially ordered vector metric spaces. Further, we provide nontrivial and comparative examples to show the effectiveness of our main results.
\end{abstract}

\section{Introduction and Preliminaries}

Cevik et al. 11 brought to the literature a notion of vector metric and proved Banach fixed point theorem 7 which is considered starting of metric fixed point theory in these spaces. Then, many authors have studied to obtain various fixed point results in context of vector metric spaces 2, 12, 16, 17, 18, 21. However, when consider the topological structure of vector metric spaces, it may not be easy to prove a result existing in the real valued metric spaces. This is an important and interesting point for the authors. Now, we state definition of Riesz space and related properties:

Let $E$ be nonempty set and $\leq$ be a relation on $\Omega$. Then, the relation $\leq$ is called partial order, if it satisfies

(o1) $\varsigma \leq \varsigma$,

2020 Mathematics Subject Classification. Primary 54H25; Secondary, 47H10, 35B15.

Keywords and phrases. Best proximity point, $\alpha$-admissible, proximal contraction, vector metric spaces.

\hakan.sahin@amasya.edu.tr

(D) 0000-0002-4671-7950.

(C)2021 Ankara University Communications Faculty of Sciences University of Ankara-Series A1 Mathematics and Statistics 
(o2) $\varsigma \leq \eta$ and $\eta \leq \varsigma$ imply $\varsigma=\eta$,

(o3) $\varsigma \leq \eta$ and $\eta \leq z$ imply $\varsigma \leq z$.

for all $\varsigma, \eta, z \in E$. Moreover, the pair $(E, \leq)$ is said to be partially ordered set. An ordered vector space is real vector space equipped with an partial order which is compatible operations of vector space, that is,

(c1) $\varsigma \leq \eta$ implies $\varsigma+z \leq \eta+z$ for all $\varsigma, \eta, z \in E$.

(c2) $\varsigma \leq \eta$ implies $\lambda \varsigma \leq \bar{\lambda} \eta$ for all positive real number $\lambda$ and all $\varsigma, \eta \in E$.

Then, ordered vector space $(E, \leq)$ is called Riesz space if it is satisfied the supremum $\varsigma \vee \eta$ and infimum $\varsigma \wedge \eta$ in $E$ for all $\varsigma, \eta \in E$. Let $(E, \leq)$ be a Riesz space. We denote positive cone of $E$ by $E_{+}$. The notation $a_{n} \downarrow a$ means that the sequence $\left\{a_{n}\right\}$ in $E$ is nonicreasing such that $\inf \left\{a_{n}: n \in \mathbb{N}\right\}=a$. If there exists a sequence $\left\{a_{n}\right\}$ in $E$ satisfying $a_{n} \downarrow 0$ such that $\left|b_{n}-b\right| \leq a_{n}$ for all $n \in \mathbb{N}$, then the sequence $\left\{b_{n}\right\}$ in $E$ is said to be order converges to $b \in E$ where the modul of any point $a$ in $E$ is defined by $|a|=a \vee(-a)$. Moreover, if there exists a sequence $\left\{a_{n}\right\}$ in $E$ satisfying $a_{n} \downarrow 0$ such that $\left|b_{n}-b_{n+p}\right| \leq a_{n}$ for all $n, p \in \mathbb{N}$, then the sequence $\left\{b_{n}\right\}$ in $E$ is called order-Cauchy sequence. A Riesz space $E$ is said to be order-Cauchy complete if every order-Cauhy sequence in $E$ is order converges to a point in $E$. A Riesz space $(E, \leq)$ is called an Archimedean if $\frac{1}{n} a \downarrow 0$ for all $a \in E_{+}$. Further, if every subset of $E$ with upper bound (lower bound) has a supremum (infimum) in $E$, then $(E, \leq)$ is said to be Dedekind complete. Note that every Dedekind complete Riesz space is an Archimedean Riesz space. For more details about Riesz spaces see 3.11 .

We continue this section with definition of a vector metric space and its related properties.

Definition 1 ( [11]). Let $\Omega$ be a nonempty set and $(E, \leq)$ be a Riesz space. Then the mapping $\rho: \Omega \times \Omega \rightarrow E$ is said to be a vector metric if it satisfies following conditions for all $\varsigma, \eta, z \in \Omega$ :

v1) $\rho(\varsigma, \eta)=0 \Leftrightarrow \varsigma=\eta$,

v2) $\rho(\varsigma, z) \leq \rho(\varsigma, \eta)+\rho(z, \eta)$.

Moreover, $(\Omega, \rho, E)$ is called vector metric space.

Definition $2(11)$. Let $(\Omega, \rho, E)$ be a vector metric space and $\left\{\varsigma_{n}\right\}$ be a sequence in $\Omega$. Then,

(i) The sequence $\left\{\varsigma_{n}\right\}$ is said to be E-converges to $\varsigma \in \Omega$, denoted by $\varsigma_{n} \stackrel{\rho, E}{\longrightarrow} \varsigma$, if there exists a sequence $\left\{a_{n}\right\}$ satisfying $a_{n} \downarrow 0$ such that $\rho\left(\varsigma_{n}, \varsigma\right) \leq a_{n}$ for all $n \in \mathbb{N}$.

(ii) The sequence $\left\{\varsigma_{n}\right\}$ is said to be E-Cauchy sequence if there exists a sequence $\left\{a_{n}\right\}$ satisfying $a_{n} \downarrow 0$ such that $\rho\left(\varsigma_{n}, \varsigma_{n+p}\right) \leq a_{n}$ for all $n, p \in \mathbb{N}$.

(iii) The vector metric space $(\Omega, \rho, E)$ is said to be $E$-complete if every $E$-Cauchy sequence in $\Omega E$-converges to a point in $\Omega$. 
Lemma 3 ( $[11)$. Let $(\Omega, \rho, E)$ be a vector metric space, $\left\{\varsigma_{n}\right\},\left\{\eta_{n}\right\}$ be sequences in $\Omega$ such that $\varsigma_{n} \stackrel{\rho, E}{\longrightarrow} \varsigma \in \Omega$ and $\eta_{n} \stackrel{\rho, E}{\rightarrow} \eta \in \Omega$. Then, we have following properties:

(i) The limits $\varsigma$ and $\eta$ are unique.

(ii) Each subsequence of $\left\{\varsigma_{n}\right\}$ E-converges to $\varsigma$.

(iii) The sequence $\left\{\rho\left(\varsigma_{n}, \eta_{n}\right)\right\}$ order converges to $\rho(\varsigma, \eta)$.

Definition $4([10])$. Let $(\Omega, \rho, E)$ and $(\Lambda, \rho, F)$ be vector metric spaces, $\left\{\varsigma_{n}\right\}$ be a sequence in $\Omega, \varsigma \in \Omega$. For a mapping $T: \Omega \rightarrow \Lambda$ if $\varsigma_{n} \stackrel{\rho, E}{\rightarrow} \varsigma$ implies $T \varsigma_{n} \stackrel{\rho, F}{\longrightarrow} T \varsigma$, then $T$ is said to be vectorially continuous at $\varsigma$. If the mapping $T$ is vectorially continuous mapping at each point in $\Omega$, then it is called vectorially continuous on $\Omega$.

On the other hand, recently, considering nonself mappings on complete metric spaces, the results proved in fixed point theory have been extended. Let $(\Omega, \rho)$ be a metric space, $M, N \subseteq \Omega$ be nonempty subsets of $\Omega$ and $T: M \rightarrow N$ be a mapping. If $M \cap N=\emptyset$, then $T$ does not have a fixed point. In this case, best proximity point theory tries to find existence of a point $\varsigma \in M$ such that $\rho(\varsigma, T \varsigma)=\rho(M, N)$ which is called a best proximity point. Note that, searching for the existence a best proximity point of the mapping $T$ is actually searching a optimal solution for the minimization problem $\min _{\varsigma \in M} \rho(\varsigma, T \varsigma)$. Further, best proximity point results become fixed point results in special case $M=N=\Omega$. Because of these reasons, best proximity point theory is one of the area attracted the most attention lately $1,4,4,5,6,9,13,15,19,20$.

In the present paper, we aim to extend some fixed point results obtained on both vector metric spaces and partially ordered vector metric spaces. Introducing two new concepts so called $\Omega$-Dedekind complete Riesz space and $\alpha$-vector proximal contraction mapping, we prove some best proximity point theorems for such mappings on vector metric spaces $(\Omega, \rho, E)$ where $(E, \leq)$ is $\Omega$-Dedekind complete Riesz space. Finally, we give nontrivial examples to support our main results.

\section{Main Results}

We start to this section with the following our new definitions which are useful in the sequel.

Definition 5. Let $(\Omega, \rho, E)$ be a vector metric space. If, for every subsets $M, N$ of $\Omega, \rho(M, N)$ is in $E$, then $(E, \leq)$ is said to be a $\Omega$-Dedekind complete Riesz space.

Note that every Dedekind complete Riesz space $(E, \leq)$ is an $\Omega$-Dedekind complete Riesz space for all $(\Omega, \rho, E)$ vector metric space. However, the converse may not be true. The following example shows this fact:

Example 6. Let $E=C[0,1]$ be the family of all continuous function from $[0,1]$ to real numbers and $\Omega=[0, \infty)$. Then, $E$ is Riesz space with the pointwise ordering 
$\leq$ but it is not Dedekind complete. Indeed, consider a sequence of functions $\left\{f_{n}\right\}$ defined by:

$$
f_{n}(\varsigma)=\left\{\begin{array}{ccc}
1 & , \quad 0 \leq \varsigma \leq \frac{1}{2}-\frac{1}{n} \\
-n\left(\varsigma-\frac{1}{2}\right) & , \quad \frac{1}{2}-\frac{1}{n}<\varsigma<\frac{1}{2} \\
0 & , & \frac{1}{2} \leq \varsigma \leq 1
\end{array}\right.
$$

Then, it can be seen that $\left\{f_{n}\right\}_{n \geq 3} \subseteq E$ and the set of functions $\left\{f_{n}: n \in \mathbb{N}\right\}$ is bounded from above with the function $\mathbf{1}:[0,1] \rightarrow \mathbb{R}$ defined as $\mathbf{1}(x)=1$ for all $x \in[0,1]$. However,

$$
\sup f_{n}=f(\varsigma)=\left\{\begin{array}{lll}
1 & , & 0 \leq \varsigma \leq \frac{1}{2} \\
0 & , & \frac{1}{2} \leq \varsigma \leq 1
\end{array} .\right.
$$

and hence $f \notin E$. Now, consider the mapping $\rho: \Omega \times \Omega \rightarrow E$ defined by

$$
\rho(\varsigma, \eta)=f_{\varsigma \eta}=\left\{\begin{array}{cc}
\mathbf{0} & , \quad \varsigma=\eta \\
\max \{\varsigma, \eta\} \mathbf{1} & , \quad \varsigma \neq \eta
\end{array}\right.
$$

for all $\varsigma, \eta \in \Omega$ where the function $\mathbf{0}:[0,1] \rightarrow \mathbb{R}$ defined as $\mathbf{0}(x)=0$ for all $x \in[0,1]$. In this case, $\rho(M, N)$ is real valued constant function and so $\rho(M, N) \in E$ for all $M, N \subseteq \Omega$. Therefore, $(E, \leq)$ is $\Omega$-Dedekind complete Riesz space.

Considering structure of vector metric spaces, we restate the definitions introduced in the frame of metric space as follows.

Definition 7. Let $(\Omega, \rho, E)$ be a vector metric space where $(E, \leq)$ is an $\Omega$-Dedekind complete Riesz space and $M, N$ be nonempty subsets of $\Omega$. Let $T: M \rightarrow N$ and $\alpha: M \times M \rightarrow[0, \infty)$ be mappings. If the mapping $T$ satisfies

$$
\left.\begin{array}{c}
\alpha(\varsigma, \eta) \geq 1 \\
\rho(u, T \varsigma)=\rho(M, N) \\
\rho(v, T \eta)=\rho(M, N)
\end{array}\right\} \Rightarrow \alpha(u, v) \geq 1
$$

for all $\varsigma, \eta, u, v \in M$, then $T$ is said to be $\alpha$-vector proximal admissible.

Definition 8. Let $(\Omega, \rho, E)$ be a vector metric space where $(E, \leq)$ is an $\Omega$-Dedekind complete Riesz space, $M, N$ be nonempty subsets of $\Omega$ and $T: M \rightarrow N$ be a mapping. If there exists $q \in[0,1)$ such that

$$
\left.\begin{array}{l}
\rho(u, T \varsigma)=\rho(M, N) \\
\rho(v, T \eta)=\rho(M, N)
\end{array}\right\} \Rightarrow \rho(u, v) \leq q \rho(\varsigma, \eta)
$$

for all $\varsigma, \eta, u, v \in M$, then $T$ is said to be vector proximal contraction.

Now, we introduce a new concept which is more general than proximal contraction mapping.

Definition 9. Let $(\Omega, \rho, E)$ be a vector metric space where $(E, \leq)$ is an $\Omega$-Dedekind complete Riesz space and $M, N$ be nonempty subsets of $\Omega$. For two mappings $T$ : 
$M \rightarrow N$ and $\alpha: M \times M \rightarrow[0, \infty)$ if there exists $q \in[0,1)$ such that

$$
\left.\begin{array}{c}
\alpha(\varsigma, \eta) \geq 1 \\
\rho(u, T \varsigma)=\rho(M, N) \\
\rho(v, T \eta)=\rho(M, N)
\end{array}\right\} \Rightarrow \rho(u, v) \leq q \rho(\varsigma, \eta)
$$

for all $u, v, \varsigma, \eta \in M$, then the mapping $T$ is said to be $\alpha$-vector proximal contraction mapping.

Let $(\Omega, \rho, E)$ be a vector metric space where $(E, \leq)$ is an $\Omega$-Dedekind complete Riesz space. We will consider the following sets in the rest of paper.

$$
M_{0}=\{\varsigma \in M: \rho(\varsigma, \eta)=\rho(M, N) \text { for some } \eta \in N\}
$$

and

$$
N_{0}=\{\eta \in N: \rho(\varsigma, \eta)=\rho(M, N) \text { for some } \varsigma \in M\}
$$

where $\rho(M, N)=\inf \{\rho(\varsigma, \eta): \varsigma \in M, \eta \in N\}$.

Theorem 10. Let $(\Omega, \rho, E)$ be an E-complete vector metric space where $(E, \leq)$ is an Archimedean and an $\Omega$-Dedekind complete Riesz space and $M, N$ be nonempty subsets of $\Omega$ where $M_{0}$ is E-closed and $M_{0} \neq \emptyset$. Let $\alpha: M \times M \rightarrow[0, \infty)$ be a mapping. Assume that $T: M \rightarrow N$ is a vectorially continuous $\alpha$-vector proximal contraction mapping satisfying following conditions:

(i) $T\left(M_{0}\right) \subseteq N_{0}$,

(ii) $T$ is an $\alpha$-vector proximal admissible,

(iii) There exist $\varsigma_{0}$ and $\varsigma_{1}$ in $M_{0}$ such that $\rho\left(\varsigma_{1}, T \varsigma_{0}\right)=\rho(M, N)$ and $\alpha\left(\varsigma_{0}, \varsigma_{1}\right) \geq$ 1.

Then $T$ has a best proximity point $\varsigma^{*}$ in $M$. Moreover, if there exists an another best proximity point $\eta^{*} \in M$ such that $\alpha\left(\varsigma^{*}, \eta^{*}\right) \geq 1$, then $\varsigma^{*}=\eta^{*}$.

Proof. From the hypothesis, there exist $\varsigma_{0}, \varsigma_{1} \in M_{0}$ such that

$$
\rho\left(\varsigma_{1}, T \varsigma_{0}\right)=\rho(M, N) \text { and } \alpha\left(\varsigma_{0}, \varsigma_{1}\right) \geq 1 .
$$

Since $T \varsigma_{1} \in T\left(M_{0}\right) \subseteq N_{0}$, there exists $\varsigma_{2} \in M_{0}$ such that

$$
\rho\left(\varsigma_{2}, T \varsigma_{1}\right)=\rho(M, N) .
$$

Since $T$ is $\alpha$-vector proximal contraction mapping, from (1) and (2), there exists $q \in[0,1)$ such that

and by condition (ii), we have

$$
\rho\left(\varsigma_{1}, \varsigma_{2}\right) \leq q \rho\left(\varsigma_{0}, \varsigma_{1}\right)
$$

$$
\alpha\left(\varsigma_{1}, \varsigma_{2}\right) \geq 1 \text {. }
$$

Continuing this process, we construct a sequence $\left\{\varsigma_{n}\right\}$ such that

$$
\begin{gathered}
\rho\left(\varsigma_{n+1}, T \varsigma_{n}\right)=\rho(M, N) \\
\rho\left(\varsigma_{n}, \varsigma_{n+1}\right) \leq q \rho\left(\varsigma_{n-1}, \varsigma_{n}\right) \leq \cdots \leq q^{n} \rho\left(\varsigma_{0}, \varsigma_{1}\right)
\end{gathered}
$$

and

$$
\alpha\left(\varsigma_{n}, \varsigma_{n+1}\right) \geq 1
$$


for all $n \in \mathbb{N}$. For arbitrary $n, p \in \mathbb{N}$

$$
\begin{aligned}
\rho\left(\varsigma_{n}, \varsigma_{n+p}\right) & \leq \rho\left(\varsigma_{n}, \varsigma_{n+1}\right)+\rho\left(\varsigma_{n+1}, \varsigma_{n+2}\right)+\cdots+\rho\left(\varsigma_{n+p-1}, \varsigma_{n+p}\right) \\
& \leq q^{n} \rho\left(\varsigma_{0}, \varsigma_{1}\right)+q^{n+1} \rho\left(\varsigma_{0}, \varsigma_{1}\right)+\cdots+q^{n+p-1} \rho\left(\varsigma_{0}, \varsigma_{1}\right) \\
& \leq\left(q^{n}+q^{n+1}+\cdots+q^{n+p-1}\right) \rho\left(\varsigma_{0}, \varsigma_{1}\right) \\
& \leq \frac{q^{n}}{1-q} \rho\left(\varsigma_{0}, \varsigma_{1}\right)
\end{aligned}
$$

which implies that $\left\{\varsigma_{n}\right\}$ is $E$-Cauchy sequence because of the fact that $(E, \leq)$ is an Archimedean Riesz space. Since $(\Omega, \rho, E)$ is $E$-complete vector metric space and $M_{0}$ is $E$-closed, there exist a sequence $\left\{a_{n}\right\} \subseteq E$ satisfying $a_{n} \downarrow 0$ and $\varsigma^{*} \in M_{0}$ such that

$$
\rho\left(\varsigma_{n}, \varsigma^{*}\right) \leq a_{n}
$$

for all $n \in \mathbb{N}$. Since $T$ is a vectorially continuous mapping on $M, T \varsigma_{n} \stackrel{\rho, E}{\rightarrow} T \varsigma^{*}$. Then, there exists a sequence $\left\{b_{n}\right\}$ satisfying $b_{n} \downarrow 0$ such that

$$
\rho\left(T \varsigma_{n}, T \varsigma^{*}\right) \leq b_{n}
$$

for all $n \in \mathbb{N}$. Hence, from (3), (5) and (6), we have

$$
\begin{aligned}
\rho\left(\varsigma^{*}, T \varsigma^{*}\right) & \leq \rho\left(\varsigma^{*}, \varsigma_{n+1}\right)+\rho\left(\varsigma_{n+1}, T \varsigma_{n}\right)+\rho\left(T \varsigma_{n}, T \varsigma^{*}\right) \\
& \leq a_{n+1}+\rho(M, N)+b_{n}
\end{aligned}
$$

which implies that

$$
0 \leq\left|\rho\left(\varsigma^{*}, T \varsigma^{*}\right)-\rho(M, N)\right| \leq a_{n+1}+b_{n}
$$

for all $n \in \mathbb{N}$. Therefore, $\varsigma^{*}$ is a best proximity point of $T$, that is,

$$
\rho\left(\varsigma^{*}, T \varsigma^{*}\right)=\rho(M, N) .
$$

To show the uniqueness of best proximity point, assume that there exists another best proximity point $\eta^{*} \in M$ which is distinct from $\varsigma^{*}$ such that

$$
\alpha\left(\varsigma^{*}, \eta^{*}\right) \geq 1 \text {. }
$$

Since $T$ is $\alpha$-vector proximal contraction mapping, from (7), (8) and $\rho\left(\eta^{*}, T \eta^{*}\right)=$ $\rho(M, N)$, we obtain

$$
\rho\left(\varsigma^{*}, \eta^{*}\right) \leq q \rho\left(\varsigma^{*}, \eta^{*}\right)
$$

which contradicts $\varsigma^{*} \neq \eta^{*}$. Then, proof is finished.

The following example shows the effectiveness of Theorem 10 because of the fact that any real valued metric can not be applied to this example.

Example 11. Let $E=\mathbb{R}^{2}$ be an Archimedean Riesz space with the componentwise ordering relation $\leq$ and the usual operations. Let $\Omega=[0, \infty) \times[0, \infty)$ and $\rho$ : $\Omega \times \Omega \rightarrow E$ be a mapping defined by

$$
\rho\left(\left(\varsigma_{1}, \varsigma_{2}\right),\left(\eta_{1}, \eta_{2}\right)\right)=\frac{1}{2}\left(\left|\varsigma_{1}-\eta_{1}\right|,\left|\varsigma_{2}-\eta_{2}\right|\right)
$$


for all $\left(\varsigma_{1}, \varsigma_{2}\right),\left(\eta_{1}, \eta_{2}\right) \in \Omega$. Then, the tripled $(\Omega, \rho, E)$ is an E-complete vector metric space and $(E, \leq)$ is an $\Omega$-Dedekind complete Riesz space. Consider the subsets of $\Omega$

$$
M=\{(\varsigma, 0): \varsigma \in \mathbb{R}\}
$$

and

$$
N=\{(\varsigma, 1): \varsigma \in \mathbb{R}\} .
$$

Then, $M_{0}=M, N_{0}=N$ and $\rho(M, N)=\left(0, \frac{1}{2}\right)$. Moreover, it can be seen that $M_{0}$ is E-closed. Define the mapping $T: M \rightarrow N$ as $T(\varsigma, 0)=\left(\frac{\varsigma}{2}, 1\right)$ for all $(\varsigma, 0) \in M$ and $\alpha: M \times M \rightarrow[0, \infty)$ as

$$
\alpha\left(\left(\varsigma_{1}, 0\right),\left(\eta_{1}, 0\right)\right)=\left\{\begin{array}{cc}
1+\left|\varsigma_{1}-\eta_{1}\right| & 0 \leq \varsigma_{1} \leq \frac{1}{2} \text { and } 0 \leq \eta_{1} \leq \frac{1}{2} \\
0 & \text { otherwise }
\end{array} .\right.
$$

Then, $T$ is a vectorially continuous and $\alpha$-vector proximal contraction mapping with $q=\frac{1}{2}$. Also, we have $T\left(M_{0}\right) \subseteq N_{0}$ and $T$ is $\alpha$-proximal admissible. Further, if we take $\varsigma=\left(\frac{1}{2}, 0\right), \eta=\left(\frac{1}{4}, 0\right)$, then it is satisfied

$$
\rho(\eta, T \varsigma)=\rho(M, N) \text { and } \alpha(\varsigma, \eta) \geq 1 .
$$

Therefore, all hypotheses of Theorem 10 are satisfied. Hence, the mapping $T$ has a unique best proximity point. On the other hand, it is clear that any real valued metric cannot applicable to this example.

Using Theorem 10 , we can obtain the following result in partially ordered vector metric spaces.

Corollary 12. Let $(\Omega, \rho, E)$ be an E-complete vector metric space where $(E, \leq)$ is an Archimedean and an $\Omega$-Dedekind complete Riesz space and $(\Omega, \lesssim)$ is a partially ordered set. Let $M, N$ be nonempty subsets of $\Omega$ where $M_{0}$ is E-closed and $M_{0} \neq \emptyset$. Assume that the mapping $T: M \rightarrow N$ is a vectorially continuous mapping on $M$ satisfying following conditions:

(i) $T\left(M_{0}\right) \subseteq N_{0}$,

(ii) It is satisfied

$$
\left.\begin{array}{l}
\rho(u, T \varsigma)=\rho(M, N) \\
\rho(v, T \eta)=\rho(M, N)
\end{array}\right\} \Rightarrow u \lesssim v \text { or } v \lesssim u
$$

for all $u, v, \varsigma, \eta \in M$ with $\varsigma \lesssim \eta$ or $\eta \lesssim \varsigma$,

(iii) There exist $\varsigma_{0}$ and $\varsigma_{1}$ in $M_{0}$ such that $\rho\left(\varsigma_{1}, T \varsigma_{0}\right)=\rho(M, N)$ and $\varsigma_{0} \lesssim \varsigma_{1}$ or $\varsigma_{1} \lesssim \varsigma_{0}$

(iv) There exists $q \in[0,1)$ such that

$$
\left.\begin{array}{l}
\rho(u, T \varsigma)=\rho(M, N) \\
\rho(v, T \eta)=\rho(M, N)
\end{array}\right\} \Rightarrow \rho(u, v) \leq q \rho(\varsigma, \eta)
$$

for all $u, v, \varsigma, \eta \in M$ with $\varsigma \lesssim \eta$ or $\eta \lesssim \varsigma$.

Then $T$ has a best proximity point $\varsigma^{*}$ in $M$. Moreover, if there exists an another best proximity point $\eta^{*} \in M$ such that $\varsigma^{*} \lesssim \eta^{*}$ or $\eta^{*} \lesssim \varsigma^{*}$, then $\varsigma^{*}=\eta^{*}$. 
Proof. If we define a mapping $\alpha: M \times M \rightarrow[0, \infty)$ as

$$
\alpha(\varsigma, \eta)=\left\{\begin{array}{cc}
1, & \varsigma \lesssim \eta \text { or } \eta \lesssim \varsigma \\
0, & \text { otherwise }
\end{array}\right.
$$

for all $\varsigma, \eta \in \Omega$, then from condition (ii) $T$ is an $\alpha$-vector proximal admissible mapping. Further, using (iv) we have that $T$ is an $\alpha$-vector proximal contraction mapping. Therefore, all hypotheses of Theorem 10 are satisfied and so $T$ has a best proximity point.

If we take $\alpha(\varsigma, \eta)=1$ in Theorem 10 , we deduce a best proximity point result as follows.

Corollary 13. Let $(\Omega, \rho, E)$ be an E-complete vector metric space where $(E, \leq)$ is an Archimedean and an $\Omega$-Dedekind complete Riesz space. Let $M, N$ be nonempty subsets of $\Omega$ where $M_{0}$ is E-closed, $M_{0} \neq \emptyset$ and $T: M \rightarrow N$ be a vectorially continuous vector proximal contraction mapping satisfying $T\left(M_{0}\right) \subseteq N_{0}$. Then, $T$ has a unique best proximity point in $M$.

In Theorem 10 , to remove vectorially continuous of the mapping $T$, we can utilize the following condition $(H)$ :

$(H)$ For every sequence $\left\{\varsigma_{n}\right\}$ in $M$ satisfying $\alpha\left(\varsigma_{n}, \varsigma_{n+1}\right) \geq 1$ for all $n \in \mathbb{N}$ and $\varsigma_{n} \stackrel{\rho, E}{\longrightarrow} \varsigma \in M$ as $n \rightarrow \infty$, there exists a subsequence $\left\{\varsigma_{n_{k}}\right\}$ of $\left\{\varsigma_{n}\right\}$ such that $\alpha\left(\varsigma_{n_{k}}, \varsigma\right) \geq 1$ for all $k \in \mathbb{N}$.

Theorem 14. Let $(\Omega, \rho, E)$ be an E-complete vector metric space where $(E, \leq)$ is an Archimedean and an $\Omega$-Dedekind complete Riesz space and $M, N$ be nonempty subsets of $\Omega$ where $M_{0}$ is E-closed and $M_{0} \neq \emptyset$. Assume that the condition $(H)$ holds. Let $\alpha: M \times M \rightarrow[0, \infty)$ be a mapping and $T: M \rightarrow N$ be an $\alpha$-vector proximal contraction mapping satisfying following conditions:

(i) $T\left(M_{0}\right) \subseteq N_{0}$,

(ii) $T$ is an $\alpha$-vector proximal admissible,

(iii) There exist $\varsigma_{0}$ and $\varsigma_{1}$ in $M_{0}$ such that $\rho\left(\varsigma_{1}, T \varsigma_{0}\right)=\rho(M, N)$ and $\alpha\left(\varsigma_{0}, \varsigma_{1}\right) \geq$ 1.

Then $T$ has a best proximity point $\varsigma^{*}$ in $M$. Moreover, if there exists an another best proximity point $\eta^{*} \in M$ such that $\alpha\left(\varsigma^{*}, \eta^{*}\right) \geq 1$, then $\varsigma^{*}=\eta^{*}$.

Proof. From the hypothesis, there exist $\varsigma_{0}, \varsigma_{1} \in M_{0}$ such that

$$
\rho\left(\varsigma_{1}, T \varsigma_{0}\right)=\rho(M, N) \text { and } \alpha\left(\varsigma_{0}, \varsigma_{1}\right) \geq 1 .
$$

Since $T \varsigma_{1} \in T\left(M_{0}\right) \subseteq N_{0}$, there exists $\varsigma_{2} \in M_{0}$ such that

$$
\rho\left(\varsigma_{2}, T \varsigma_{1}\right)=\rho(M, N) .
$$

Since $T$ is $\alpha$-vector proximal contraction mapping, from $(9)$ and 10 there exists $q \in[0,1)$ such that

$$
\rho\left(\varsigma_{1}, \varsigma_{2}\right) \leq q \rho\left(\varsigma_{0}, \varsigma_{1}\right)
$$


and bycondition (ii), we have

$$
\alpha\left(\varsigma_{1}, \varsigma_{2}\right) \geq 1
$$

Continuing this process, we construct a sequence $\left\{\varsigma_{n}\right\}$ such that

$$
\begin{gathered}
\rho\left(\varsigma_{n+1}, T \varsigma_{n}\right)=\rho(M, N) \\
\rho\left(\varsigma_{n}, \varsigma_{n+1}\right) \leq q \rho\left(\varsigma_{n-1}, \varsigma_{n}\right)
\end{gathered}
$$

and

$$
\alpha\left(\varsigma_{n}, \varsigma_{n+1}\right) \geq 1
$$

for all $n \in \mathbb{N}$. For arbitrary $n, p \in \mathbb{N}$, it can be seen that

$$
\rho\left(\varsigma_{n}, \varsigma_{n+p}\right) \leq \frac{q^{n}}{1-q} \rho\left(\varsigma_{0}, \varsigma_{1}\right)
$$

which implies that $\left\{\varsigma_{n}\right\}$ is $E$-Cauchy sequence because of the fact that $(E, \leq)$ is an Archimedean Riesz space. Since $(\Omega, \rho, E)$ is $E$ - complete vector metric space and $M_{0}$ is $E$-closed, there exists a sequence $\left\{a_{n}\right\}$ satisfying $a_{n} \downarrow 0$ and $\varsigma^{*} \in M_{0}$ such that

$$
\rho\left(\varsigma_{n}, \varsigma^{*}\right) \leq a_{n}
$$

for all $n \in \mathbb{N}$. Since $T \varsigma^{*} \in T\left(M_{0}\right) \subset N_{0}$, there exists $z^{*} \in M_{0}$ such that

$$
\rho\left(z^{*}, T \varsigma^{*}\right)=\rho(M, N) .
$$

On the other hand, from 120,130 and the condition $(H)$ there exists a subsequence $\left\{\varsigma_{n_{k}}\right\}$ of $\left\{\varsigma_{n}\right\}$ such that $\alpha\left(\varsigma_{n_{k}}, \varsigma^{*}\right) \geq 1$ for all $k \in \mathbb{N}$. Because of (11) and (14), we get

$$
\begin{aligned}
\rho\left(\varsigma_{n_{k}+1}, z^{*}\right) & \leq q \rho\left(\varsigma_{n_{k}}, \varsigma^{*}\right) \\
& \leq q a_{n_{k}}
\end{aligned}
$$

for all $k \in \mathbb{N}$ which means $\varsigma_{n_{k}+1} \stackrel{\rho, E}{\rightarrow} z^{*}$. Because of the fact that the limit of $\left\{\varsigma_{n_{k}+1}\right\}$ is unique, it is obtained that $\varsigma^{*}=z^{*}$. Therefore, from (14), $\varsigma^{*}$ is a best proximity point of $T$. It can be shown the uniqueness of best proximity point as in Theorem 10

The following example is important to show the difference between Theorem 10 and Theorem 14 .

Example 15. Let $E=\mathbb{R}^{2}$ be an Archimedean Riesz space with the componentwise ordering relation $\leq$ and the usual operations. Let $\Omega=[0, \infty) \times[0, \infty)$ and $\rho$ : $\Omega \times \Omega \rightarrow E$ be a mapping defined by

$$
\rho\left(\left(\varsigma_{1}, \varsigma_{2}\right),\left(\eta_{1}, \eta_{2}\right)\right)=\left(\frac{1}{2}\left|\varsigma_{1}-\eta_{1}\right|, \frac{1}{3}\left|\varsigma_{2}-\eta_{2}\right|\right)
$$

for all $\left(\varsigma_{1}, \varsigma_{2}\right),\left(\eta_{1}, \eta_{2}\right) \in \Omega$. Then, the tripled $(\Omega, \rho, E)$ is an E-complete vector metric space and $(E, \leq)$ is an $\Omega$-Dedekind complete Riesz space. Consider the sets

$$
M=\{(\varsigma, 0): \varsigma \geq 0, \varsigma \in \mathbb{R}\}
$$


and

$$
N=\{(\varsigma, 1): \varsigma \geq 0, \varsigma \in \mathbb{R}\}
$$

Then, $M_{0}=M, N_{0}=N$ and $\rho(M, N)=\left(0, \frac{1}{3}\right)$. Moreover, it can be easily seen that $M_{0}$ is E-closed. Define the mapping $T: M \rightarrow N$ as

$$
T(\varsigma, 0)=\left\{\begin{array}{lll}
\left(\frac{\varsigma}{3}, 1\right) & , \quad \varsigma<\frac{1}{2} \\
\left(\frac{\varsigma}{2}, 1\right) & , \quad \varsigma \geq \frac{1}{2}
\end{array}\right.
$$

for all $(\varsigma, 0) \in M$ and $\alpha: M \times M \rightarrow[0, \infty)$ as

$$
\alpha\left(\left(\varsigma_{1}, 0\right),\left(\eta_{1}, 0\right)\right)=\left\{\begin{array}{cc}
1+\left|\varsigma_{1}-\eta_{1}\right| & , \quad 0 \leq \varsigma_{1} \leq \frac{1}{3} \text { and } 0 \leq \eta_{1} \leq \frac{1}{3} \\
0 & \text { otherwise }
\end{array} .\right.
$$

Then, $T$ is an $\alpha$-vector proximal contraction mapping with $q=\frac{1}{3}$. However, although $T$ is not a vectorially continuous mapping, the condition $(H)$ holds. Indeed, if we consider the sequence $\left(\varsigma_{n}\right)=\left(\frac{1}{2}-\frac{1}{n}, 0\right)_{n \in \mathbb{N}}$, then $\varsigma_{n} \stackrel{\rho, E}{\longrightarrow}\left(\frac{1}{2}, 0\right)$. But, the sequence $\left(T \varsigma_{n}\right)=\left(\frac{1}{6}-\frac{1}{3 n}, 1\right)_{n \in \mathbb{N}}$ is not E-convergent to $T \varsigma=\left(\frac{1}{4}, 1\right)$. Also, we have $T\left(M_{0}\right) \subseteq N_{0}$ and $T$ is $\alpha$-vector proximal admissible. Further, if we take $\varsigma=\left(\frac{1}{3}, 0\right)$, $\eta=\left(\frac{1}{9}, 0\right)$, then it is satisfied

$$
\rho(\eta, T \varsigma)=\rho(M, N) \text { and } \alpha(\varsigma, \eta) \geq 1 .
$$

Therefore, all hypotheses of Theorem 14 are satisfied. Hence, the mapping $T$ has a best proximity point. On the other hand, since the mapping $T$ is not vectorially continuous, Theorem 10 cannot be applied to this example.

Using Theorem 14, we can obtain the following result in partially ordered vector metric spaces.

Corollary 16. Let $(\Omega, \rho, E)$ be an E-complete vector metric space where $(E, \leq)$ is an Archimedean and an $\Omega$-Dedekind complete Riesz space and $M, N$ be nonempty subsets of $\Omega$ where $M_{0}$ is E-closed and $M_{0} \neq \emptyset$. Let $T: M \rightarrow N$ be a mapping satisfying following conditions:

(i) $T\left(M_{0}\right) \subseteq N_{0}$,

(ii) It is satisfied

$$
\left.\begin{array}{l}
\rho(u, T \varsigma)=\rho(M, N) \\
\rho(v, T \eta)=\rho(M, N)
\end{array}\right\} \Rightarrow u \lesssim v \text { or } v \lesssim u
$$

for all $u, v, \varsigma, \eta \in M$ with $\varsigma \lesssim \eta$ or $\eta \lesssim \varsigma$,

(iii) There exist $\varsigma_{0}$ and $\varsigma_{1}$ in $M_{0}$ such that $\rho\left(\varsigma_{1}, T \varsigma_{0}\right)=\rho(M, N)$ and $\varsigma_{0} \lesssim \varsigma_{1}$ or $\varsigma_{1} \lesssim \varsigma_{0}$,

(iv) There exists $q \in[0,1)$ such that

$$
\left.\begin{array}{l}
\rho(u, T \varsigma)=\rho(M, N) \\
\rho(v, T \eta)=\rho(M, N)
\end{array}\right\} \Rightarrow \rho(u, v) \leq q \rho(\varsigma, \eta)
$$

for all $u, v, \varsigma, \eta \in M$ with $\varsigma \lesssim \eta$ or $\eta \lesssim \varsigma$, 
(v) for every sequence $\left\{\varsigma_{n}\right\}$ in $M$ satisfying $\varsigma_{n} \lesssim \varsigma_{n+1}$ or $\varsigma_{n+1} \lesssim \varsigma_{n}$ for all $n \in \mathbb{N}$ and $\varsigma_{n} \stackrel{\rho, E}{\longrightarrow} \varsigma \in M$ as $n \rightarrow \infty$, there exists a subsequence $\left\{\varsigma_{n_{k}}\right\}$ of $\left\{\varsigma_{n}\right\}$ such that $\varsigma_{n_{k}} \lesssim \varsigma$ or $\varsigma \lesssim \varsigma_{n_{k}}$ for all $k \in \mathbb{N}$.

Then, $T$ has a best proximity point $\varsigma^{*}$ in $M$. Moreover, if there exists an another best proximty point $\eta^{*} \in M$ such that $\varsigma^{*} \lesssim \eta^{*}$ or $\eta^{*} \lesssim \varsigma^{*}$, then $\varsigma^{*}=\eta^{*}$.

If we take $\alpha(\varsigma, \eta)=1$ in Theorem 14 , we deduce the following best proximity point result for the vector proximal contraction mappings.

Corollary 17. Let $(\Omega, \rho, E)$ be an E-complete vector metric space where $(E, \leq)$ is an Archimedean and an $\Omega$-Dedekind complete Riesz space and $M, N$ be nonempty subsets of $\Omega$ where $M_{0}$ is E-closed and $M_{0} \neq \emptyset$. Let $T: M \rightarrow N$ be a vector proximal contraction mapping satisfying $T\left(M_{0}\right) \subseteq N_{0}$. Then, $T$ has a unique best proximity point $\varsigma^{*}$ in $M$.

Taking $M=N=\Omega$ in Theorem 10 and Theorem 14 , we can obtain the following fixed point result in vector metric spaces.

Corollary 18. Let $(\Omega, \rho, E)$ be an E-complete vector metric space where $(E, \leq)$ is an Archimedean and an $\Omega$-Dedekind complete Riesz space. Assume that $\alpha: \Omega \times \Omega \rightarrow$ $[0, \infty)$ and $T: \Omega \rightarrow \Omega$ are mappings satisfying following conditions:

(i) $T$ is $\alpha$-admissible,

(ii) There exist $\varsigma_{0}$ in $\Omega$ such that $\alpha\left(\varsigma_{0}, T \varsigma_{0}\right) \geq 1$,

(iii) $T$ is a vectorially continuous or condition $(H)$ holds,

If there exists $q \in[0,1)$ such that

$$
\rho(T \varsigma, T \eta) \leq q \rho(\varsigma, \eta)
$$

for all $\varsigma, \eta \in \Omega$ with $\alpha(\varsigma, \eta) \geq 1$, then $T$ has a fixed point $\varsigma^{*}$ in $\Omega$. Moreover, if there exists an another fixed point $\eta^{*} \in \Omega$ such that $\alpha\left(\varsigma^{*}, \eta^{*}\right) \geq 1$, then $\varsigma^{*}=\eta^{*}$.

Taking $M=N=\Omega$ in Corollary 12 and Corollary 16 , we can obtain the following fixed point result in vector metric spaces which is the main result of 12 .

Corollary 19. Let $(\Omega, \rho, E)$ be an E-complete vector metric space where $(E, \leq)$ is an Archimedean and an $\Omega$-Dedekind complete Riesz space and $(\Omega, \lesssim)$ is a partially ordered set. Assume that $T: \Omega \rightarrow \Omega$ is a mapping satisfying following conditions:

(i) $\varsigma \lesssim \eta$ or $\eta \lesssim \varsigma$ implies $T \varsigma \lesssim T \eta$ or $T \eta \lesssim T \varsigma$ for all $\varsigma, \eta \in \Omega$,

(ii) There exists $\varsigma_{0} \in \Omega$ such that $\varsigma_{0} \lesssim T \varsigma_{0}$ or $T \varsigma_{0} \lesssim \varsigma_{0}$,

(iii) There exists $q \in[0,1)$ such that

$$
\rho(T \varsigma, T \eta) \leq q \rho(\varsigma, \eta)
$$

for all $\varsigma, \eta \in \Omega$ with $\varsigma \lesssim \eta$ or $\eta \lesssim \varsigma$,

(iv) $T$ is a vectorially continuous or for every sequence $\left\{\varsigma_{n}\right\}$ in $\Omega$ satisfying $\varsigma_{n} \lesssim \varsigma_{n+1}$ or $\varsigma_{n+1} \lesssim \varsigma_{n}$ for all $n \in \mathbb{N}$ and $\varsigma_{n} \stackrel{\rho, E}{\rightarrow} \varsigma \in \Omega$ as $n \rightarrow \infty$, there exists a subsequence $\left\{\varsigma_{n_{k}}\right\}$ of $\left\{\varsigma_{n}\right\}$ such that $\varsigma_{n_{k}} \lesssim \varsigma$ or $\varsigma \lesssim \varsigma_{n_{k}}$ for all $k \in \mathbb{N}$. 
Then, $T$ has a fixed point $\varsigma^{*}$ in $\Omega$. Moreover, if there exists an another fixed point $\eta^{*} \in \Omega$ such that $\varsigma^{*} \lesssim \eta^{*}$ or $\eta^{*} \lesssim \varsigma^{*}$, then $\varsigma^{*}=\eta^{*}$.

\section{Conclusion}

In this paper, we first give a new definition of $\Omega$-Dedekind complete Riesz space $(E, \leq)$ on a vector metric space $(\Omega, \rho, E)$, and so we obtain a new family of Riesz space which is a larger than the class of Dedekind complete Riesz space in the frame of vector metric space. We also introduce a new notion so called $\alpha$-vector proximal contraction mapping including the concept of $\alpha$-proximal contraction defined Samet et al. [14]. Then, for the first time, we obtain some best proximity point results on vector metric spaces $(\Omega, \rho, E)$ where $(E, \leq)$ is $\Omega$-Dedekind complete Riesz space and hence we extend some fixed point results proved on both vector metric spaces and partially ordered vector metric spaces such as 7, 8, 12.

Declaration of Competing Interests The authors declare that they have no known competing financial interest or personal relationships that could have appeared to influence the work reported in this paper.

Acknowledgement The authors are thankful to the referee for making valuable suggestions leading to the better presentations of the paper.

\section{REFERENCES}

[1] Alghamdi, M. A., Shahzad, N., Vetro F., Best proximity points for some classes of proximal contractions, Abstract and Applied Analysis, 2013 (2013), Article ID: 713252.

[2] Ali, M. U., Bejenaru, A., Kamran, T., The order-convergence of the Thakur iterative process for Hardy-Rogers contractions in order-Banach spaces, Journal of Mathematical Analysis, 9(4) (2018), 61-74.

[3] Aliprantis, C. D., Border, K. C., Infinite Dimensional Analysis, Berlin, Springer-Verlag, 1999.

[4] Altun, I., Aslantas, M., Sahin, H., Best proximity point results for $p$-proximal contractions, Acta Math. Hungar., 162 (2020), 393-402.

[5] Aslantas, M., Sahin, H., Altun, I., Best proximity point theorems for cyclic $p$-contractions with some consequences and applications, Nonlinear Analysis Modelling and Control, 26 (1) (2021), 113-129.

[6] Aydi, H., Lakzian, H., Mitrović, Z. D., Radenović, S., Best proximity points of $M T$-cyclic contractions with property UC, Numerical Functional Analysis and Optimization, 41 (2020), $1-12$.

[7] Banach, S., Sur les opérations dans les ensembles abstraits et leur applications aux équations intégrales, Fund. Math., 3 (1922), 133-181.

[8] Basha, S. S., Extensions of Banach's contraction principle, Numer. Funct. Anal. Optim., 31 (5) (2010), 569-576.

[9] Basha, S. S., Shahzad, N., Vetro, C., Best proximity point theorems for proximal cyclic contractions, Journal of Fixed Point Theory and Applications, 19 (4) (2017), 2647-2661.

[10] Çevik, C., On continuity functions between vector metric spaces, Journal of Function Spaces, (2014), Article ID 753969.

[11] Çevik, C., Altun, I., Vector metric spaces and some properties, Topol. Methods Nonlinear Anal., 34 (2009), 375-382.

[12] Çevik, C., Altun, I., Sahin, H., Ozeken, C. C., Some fixed point theorems for contractive mapping in ordered vector metric spaces, J. Nonlinear Sci. Appl., 10(4) (2017), 1424-1432. 
[13] Eldred, A. A., Veeramani, P., Existence and convergence of best proximity points, J. Math. Anal. Appl., 323 (2006), 1001-1006.

[14] Jleli, M., Samet, B., Best proximity points for $\alpha$ - $\psi$-proximal contractive type mappings and application, Bull. Sci. Math., 137 (2013), 977-995.

[15] Nashine, H. K., Kumam, P., Vetro, C., Best proximity point theorems for rational proximal contractions, Fixed Point Theory and Applications, 2013 (2013), Article ID: 95.

[16] Ozeken, C., Cevik, C., Unbounded vectorial Cauchy completion of vector metric spaces, Gazi University Journal of Science, 33(3) (2020), 761-765.

[17] Páles, Zs., Petre, I. R., Iterative fixed point theorems in E-metric spaces, Acta Math. Hung., 140(2013), 134-144.

[18] Rahimi, H., Abbas, M., Rad, G., Common fixed point results for four mappings on ordered vector metric spaces, Filomat, 29(4) (2015), 865-878.

[19] Sahin, H., Aslantas, M., Altun, I., Feng-Liu type approach to best proximity point results for multivalued mappings, Journal of Fixed Point Theory and Applications, 22(1) (2020), Doi: 10.1007/s11784-019-0740-9.

[20] Sankar Raj, V., A best proximity point theorem for weakly contractive non-self-mappings, Nonlinear Anal., 74 (2011), 4804-4808.

[21] Soleimani Rad, G., Altun, I., Common fixed point results on vector metric spaces, Journal of Linear and Topological Algebra (JLTA), 5(1) (2016), 29-39. 